\title{
Théologiques
}

\section{Enchevêtrés dans des histoires de pouvoir}

\section{Jean-Guy Nadeau}

Volume 8, numéro 2, automne 2000

Le pouvoir

URI : https://id.erudit.org/iderudit/005019ar

DOI : https://doi.org/10.7202/005019ar

Aller au sommaire du numéro

Éditeur(s)

Faculté de théologie de l'Université de Montréal

ISSN

1188-7109 (imprimé)

1492-1413 (numérique)

Découvrir la revue

Citer ce document

Nadeau, J.-G. (2000). Enchevêtrés dans des histoires de pouvoir. Théologiques, 8(2), 3-13. https://doi.org/10.7202/005019ar d'utilisation que vous pouvez consulter en ligne.

https://apropos.erudit.org/fr/usagers/politique-dutilisation/ 


\section{Enchevêtrés dans des histoires de pouvoir}

Jean-Guy NADEAU

Nous sommes enchevêtrés dans des histoires, écrivait Wilhelm Schapp, des histoires à travers lesquelles se construisent l'identité de l'individu comme celle de la nation. Or, ces histoires sont des histoires de pouvoir, de coopération, de lutte, de domination, de résistance, d'abus, de survie. Et ce, jusque dans la dite histoire sainte dont les récits n'ont rien d'histoires pieuses mais sont, comme le notait Robert Alter, des histoires de ruses, de meurtres, de viols, de guerres, de massacres ${ }^{1}$. Et de salut. Nos histoires, comme celles de la Bible, sont dramatiques : un homme tue son frère, les premiers nés d'un peuple sont exterminés, des femmes et des bébés sont passés au fil de l'épée, des industriels exploitent le travail de leurs ouvriers et ouvrières, un homme abuse de sa fille ou un jeune gardien du bébé dont il a la charge, une communauté impose sa religion et ses violences à l'ensemble d'un pays, une Américaine dans la quarantaine électrocute un inconnu dans le cadre d'une expérience sur la torture ${ }^{2}$ - un usage du pouvoir que $25 \%$ des Français jugent " acceptable dans certains cas exceptionnels " dont la définition semble plutôt large ${ }^{3}$ - un prêtre remet les péchés, une femme guérie se relève,

1. Paul Ricoeur, "Le récit interprétatif. Exégèse et Théologie dans les récits de la Passion. » RSR 73/1 (1985) 18.

2. Stanley Milgram, Obedience to Authority, Harper \& Row, 1974, pp 79 ss, cité dans John Conroy, Unspeakable Acts, Ordinary People. The Dynamics of Torture, Alfred Knopf, New York, 2000, p. 98.

3. Connaissance, attitude et jugement des français sur la torture, sondage CSA / Amnesty International-Le Monde, septembre 2000. Disponible sur le site http://www.amnesty.asso.fr/02_agir/24_campagnes/torture/Sondage.htm 
une autre décroche un emploi après des années de formation, un homme dans la quarantaine apprend à écrire, un autre peut marcher après des mois de thérapie, une femme confronte son agresseur, de jeunes ouvriers luttent pour fonder un syndicat chez McDonald's.

Dans ces histoires qui tissent notre existence, nous sommes constamment confrontés au pouvoir que nous exerçons et que nous devons exercer pour vivre, confrontés au pouvoir que les autres exercent, aux limites, aux abus et aux risques d'abus de notre pouvoir et de celui des autres. C'est ainsi que le pouvoir constitue un objet majeur des sciences humaines et sociales, au point que Bertrand Russell y trouvait un concept aussi fondamental dans les sciences sociales que l'énergie en physique.

Or, le pouvoir apparaît le plus souvent comme un problème, un problème dont les deux faces, l'abus et le manque, sont souvent l'envers l'une de l'autre. Si le pouvoir-sur est largement récusé, surtout dans les milieux de gauche, le pouvoir-de (la puissance?) est de plus en plus réclamé par des individus, des collectivités, voire des peuples entiers ${ }^{4}$. À ce double problème, s'ajoute, particulièrement dans le discours religieux, celui de légitimations du pouvoir, elles-mêmes souvent abusives. Comme le sacré qu'il détermine souvent, le pouvoir fascine et effraie, particulièrement quand il ne connaît pas ou presque pas de limite. D'où, entre autres raisons, la sacralisation de certains phénomènes naturels ou politiques doués d'une puissance ou d'un pouvoir particulier, ou particulièrement évident, et sur lesquels se sont adossés, comme sur leur archéologie, maints pouvoirs religieux. Nous y reviendrons.

Dans la littérature scientifique, le pouvoir apparaît d'abord, et de façon massive, comme pouvoir-sur, pouvoir des uns sur les autres, domination de groupes sur d'autres groupes : des chefs sur la masse, des seigneurs sur les serfs, des hommes sur les femmes, du magistère sur les fidèles, etc. Le pouvoir désigne alors la capacité de modifier ou d'influencer la conduite d'autres individus ou groupes selon sa propre volonté. C'est ainsi que Michel Foucault signale que « Le trait distinctif du pouvoir, c'est que certains hommes peuvent plus ou moins entièrement déterminer la conduite d'autres hommes - mais jamais de

4. Nous reviendrons à cette distinction, en partie injustifiée, entre pouvoirde et pouvoir-sur. 
manière exhaustive et coercitive ", ajoute Foucault pour distinguer pouvoir et force ${ }^{5}$. Le paradigme socio-politique s'avère alors dominant et le pouvoir, souvent attribué à des organisations et à des institutions, nomme un vecteur, vertical, des rapports sociaux. Le pouvoir est alors déterminé par ses effets sur l'autre et ses typologies surtout élaborées en fonction des mécanismes et des ressources de la détermination de l'autre.

D'autres types d'études, auxquelles réfère l'article de Ruard Ganzevoort, considèrent le pouvoir selon un paradigme plus horizontal, tout en étant sensibles à sa dissymétrie. Ce paradigme paraît plus près d'une sociologie de l'expérience, des théories de l'action (sociale), de l'organisation, de la communication, de la pragmatique. Erhard Friedberg, spécialiste de l'action organisationnelle, présente « le pouvoir comme capacité de structurer l'échange négocié de comportements en sa faveur [...] comme l'échange déséquilibré de possibilités d'action, c'est-à-dire de comportements entre un ensemble d'acteurs individuels et/ou collectifs ${ }^{6} »$.

De telles définitions du pouvoir mettent l'accent sur sa nature relationnelle et son caractère contextuel. «Le pouvoir, poursuit Friedberg, n'est pas un attribut, et il ne peut être possédé [... comme un bien... ]; il se trouve dans les structures préexistantes de l'espace d'action, ou plutôt dans l'asymétrie des ressources que les acteurs peuvent tirer de celles-ci pour mener leurs transactions ${ }^{7}$ ». Le pouvoir s'avère en somme attribut d'une relation et largement déterminé par l'asymétrie des ressources.

\section{Je peux, donc je suis}

Le pouvoir, cependant, et nous le voyons clairement chez Giddens, n'est pas d'abord domination sur les autres. Giddens n'élabore pas une théorie du pouvoir au sens classique du terme. C'est à travers l'étude de l'action sociale qu'il en vient à s'intéresser au pouvoir qu'il saisit comme la capacité des agents de modifier quelque chose dans le

5. Michel Foucault, "Omnes et singulatim : Vers une critique de la raison politique ", Le Débat 41 (sept-nov 1986) 34.

6. Erhard FRIEDBERG, Le pouvoir et la règle, Paris, Seuil, 1993, p. 113.

7. Ibid., p. 113-114. 
cours des événements, de mobiliser des ressources pour réaliser leur volonté ${ }^{8}$. Dans cette perspective plus proche d'Aristote que de Marx, le pouvoir apparaît pouvoir de, pouvoir d'agir, pouvoir de se déterminer, capacité, liberté, autonomie, voire responsabilité.

On ne saurait toutefois renvoyer dos-à-dos les conceptions politiques et phénoménologiques du pouvoir, ou encore ses dimensions systémiques et subjectives. D'abord, parce que le pouvoir-de n'existe pas sans rapport au social, sans enracinement social. Ensuite, parce que l'exercice du pouvoir consiste justement dans l'articulation du systémique et de l'intentionnalité de l'agent. Avec von Wright, Ricoeur identifie cette articulation comme relevant de la capacité d'un agent de faire coïncider une des choses qu'il sait pouvoir faire avec l'état initial et les relations internes de conditionnalité d'un système 9 . Comme le pouvoir chez Giddens, l'initiative ou l'intervention chez Ricoeur " cause effectivement des changements dans le monde ${ }^{10}$ ".

Paul Ricoeur, particulièrement dans Soi-même comme un autre, a fait beaucoup pour rappeler à l'anthropologie philosophique l'importance fondamentale des pouvoirs ou des capacités de base, des capacités fondatrices du sujet. S'appuyant entre autres sur la pragmatique du langage, la théorie de l'action, la phénoménologie, le récit et l'éthique, Ricoeur thématise ce que le pouvoir a d'essentiel pour l'être humain. Pouvoir dire et se dire, pouvoir agir et déterminer le monde, pouvoir raconter et interpréter (dire et faire le sens), pouvoir estimer, juger et viser le bien, autant de compétences de base nécessaires à l'élaboration du sujet. Et bien que Ricoeur n'en parle pas, on connaît les drames liés à l'absence ou à la confiscation de ces pouvoirs. À revers des capacités de base énumérées ci-dessus, se dessinent ce qu'on pourrait identifier comme des incapacités de base : incapacité de dire et de se dire ; incapacité d'agir, d'interpréter, d'évaluer ou de juger ; incapacité de saisir l'unité de sa vie ou d'établir son identité ; incapa-

8. L'Ancien Testament utilise un même terme pour désigner le pouvoir et la main.

9. Paul Ricoeur, "L'initiative ", dans Du texte à l'action, Paris, Seuil, 1986, p. 270-271.

10. Paul RicoeUR, "Approches de la personne ", Esprit 160 (mars-avril 1990) 125. 
cité de s'estimer et de se respecter aussi bien que d'estimer et de respecter autrui ${ }^{11}$.

Le pouvoir relève alors du Je peux, c'est-à-dire des capacités et de l'autonomie du sujet, davantage que de la capacité de déterminer la conduite d'autres sujets, bien que cette capacité ne lui soit pas étrangère. Le pouvoir, en effet, s'exerce toujours "sur " : sur soi, sur le monde, sur les autres. Tout se passe alors comme si nous assistions à une réhabilitation du pouvoir. Le pouvoir est considéré comme nécessaire et ce sont ses conditions d'exercice qui sont maintenant évaluées et critiquées.

D'où l'attention que Ricoeur apporte lui aussi à la dissymétrie foncière du pouvoir et de l'action : " Agir pour un agent c'est exercer un pouvoir-sur un autre agent [...] l'action est faite par quelqu'un et subie par quelqu'un d'autre ${ }^{12}$. L'action et le pouvoir appellent ainsi une éthique dont l'enjeu est que l'autre, le patient de mon action, ne devienne la victime de mon action. "Il y a moralité, poursuivait Ricoeur, parce qu'il y a violence ${ }^{13}$ ". L'abus de pouvoir de l'un, en effet, signe le plus souvent la perte de pouvoir de l'autre, perte qui peut être plus ou moins radicale. Dans certains cas, ces pertes exigent une stratégie d'appropriation ou de réappropriation de ses pouvoirs (empowerment) qui permette au sujet de retrouver ses capacités de base telles dire, agir, raconter, estimer et juger ; en somme, sa capacité de vivre, de bien vivre. Le pouvoir n'est-il pas, en fin de compte, " the ability to make or establish a claim on life [...] power is co-extensive with life itself. [...] To be alive is to exercise power in some degree ${ }^{14}$ ».

11. Cf. Jean-Guy NADEAU, "Herméneutique du sujet et théologie pratique. Quelques apports de Paul Ricoeur » dans J.C. PETIT et J.C. BRETON, dir., Seul ou avec les autres? Le salut chrétien à l'épreuve de la solidarité, Montréal, Fides, 1992, pp 359-377.

12. Paul RicOeUR, "Approches de la personne ", loc.cit., p. 126.

13. Paul Ricoeur, "Les structures téléologique et déontologique de l'action: Aristote et/ou Kant? ", dans J.-G. NADEAU, dir., L'interprétation, un défi de l'action pastorale, Montréal, Fides, 1989, p. 22.

14. James Newton Poling, The Abuse of Power. A Theological Problem, Nashville, Abigdon, 1991, p 24. Poling emprunte ici la définition de Bernard Loomer. Voir aussi James N. POLING, Deliver Us from Evil. Resisting Racial and Gender Oppression, Minneapolis, Fortress Press, 1996. 


\section{Pouvoir et religion}

La religion, on le sait, peut être utilisée aussi bien pour accroître que pour réduire le pouvoir des uns et des autres, plus souvent, en fait, les uns que les autres. Ses pouvoirs sont nombreux ${ }^{15}$. La religion exorcise et administre l'angoisse de la mort en affirmant l'immortalité de l'âme. Elle peut faciliter la survie de la collectivité par la conversion symbolique de la violence, ou elle peut elle-même justifier ou engendrer la violence. Mais surtout, la religion et plus précisément les autorités religieuses contrôlent l'accès au Sacré ou au divin, détenant ainsi une ressource majeure pour déterminer la conduite d'autres hommes (Foucault). C'est ainsi, comme on l'a déjà dit, qu'elle est souvent utilisée pour fonder le pouvoir des uns sur les autres. La théologie contemporaine est très sensible à ces fonctions sociales de la religion et dénonce régulièrement les légitimations religieuses ou l'absolutisation du pouvoir au nom du Sacré. On songe d'emblée ici aux théologies féministes et aux théologies de la libération, mais la critique théologique du pouvoir déborde largement ces théologies plus connues pour s'étendre à l'ensemble du discours théologique.

Le pouvoir vertical occupe une place majeure dans la majorité des religions où l'obéissance et la soumission apparaissent comme des vertus fondamentales. En témoigne, dans notre histoire, le Catéchisme des Provinces ecclésiastiques de Québec, Montréal et Ottawa (1888) qui, au moins jusqu'en 1951, a formé l'identité et les consciences religieuses de plusieurs de nos concitoyens ${ }^{16}$. Sa dynamique de fond était en principe celle de la foi, de l'espérance et de la charité, mais le moral organisé autour des catégories d'obligation, d'obéissance et de soumission - y avait largement envahi le théologal. 130 questions sur 508 , soit plus de $25 \%$, portaient explicitement et formellement sur une obligation. À 389 reprises, on y proposait une morale du devoir, de l'obligation, de la nécessaire soumission à un ordre donné. Le modèle en est celui de l'autorité des parents sur les enfants : c'est en obéissant

15. Jacqueline Russ, Les théories du pouvoir, Paris, Librairie générale française (Le Livre de poche), 1994, p 236ss.

16. J'emprunte ce qui suit à Gabriel DussaulT, S.J., "La religion de l'ordre... et après?", Relations 377 (1972) 330-334. Cf. aussi Gilles RAYMOND, "Quelles catéchèses? Quelles éthiques?", Communauté chrétienne 132 (1983) 580-595. 
aux commandements de Dieu et de l'Église que l'on fera le bien et évitera le mal. Cette dynamique marquait même l'énoncé des objectifs de l'Université qui " emploiera toute son énergie, toutes ses forces, à former des élèves en qui brilleront la foi, la soumission, la pureté, l'amour du travail, toutes ces vertus qui répandent une grâce exquise sur les rapports habituels de ceux qui commandent et de ceux qui obéissent, de ceux qui enseignent et de ceux qui sont étudiants ${ }^{17}$ ». De toute évidence, ce n'est pas seulement la métaphysique chrétienne qui fait problème dans notre contexte de (post-) modernité, mais aussi, et c'est bien connu, la vision et la gestion ecclésiales de l'autorité.

L'autorité des parents sur les enfants était la métaphore de base du Petit catéchisme. C'eut tout aussi bien pu être le pouvoir du pasteur sur ses brebis, pouvoir pastoral que Michel Foucault situait à l'origine de la police moderne, puis de l'État Providence qu'il considérait comme sécularisation de la pastorale chrétienne. Attention, cependant, la police ne désigne pas ici l'institution de contrôle du crime que nous connaissons, mais une technique de gouvernement qui " veille à tout ce qui touche au bonheur des hommes ${ }^{18}$ et donc à la qualité — y compris morale — de la vie, jusqu'au dessin des paquets de cigarettes canadiens. Alors que le pouvoir pastoral hébraïque supposait " une attention individuelle à chaque membre du troupeau » dont il vise le bien, la tradition chrétienne accentuera la soumission personnelle, la "dépendance individuelle et complète " envers la volonté du pasteur ${ }^{19}$. «Etre guidé était un état et vous étiez fatalement perdu si vous tentiez d'y échapper ${ }^{20}$ ». Ce à quoi font encore écho le discours et la pratique de certains pasteurs, gourous et ayatollas contemporains.

L'autorité religieuse affirme et définit d'en haut, sans appel. C'est ainsi que l'étude de la religion jette une lumière crue sur la verticalité de certains pouvoirs de définition de la vérité ou même du réel. Déjà en

17. La Semaine religieuse de Québec, vol. XII (1899-1900) 735; cité dans Dussault, p. 331.

18. Michel FOUCAULT, " Omnes et singulatim : Vers une critique de la raison politique ", conférence à l'Université de Stanford octobre 1979, Le Débat 41 (sept-nov 1986) 30. Voir aussi Michel FouCAult «Pourquoi étudier le pouvoir : la question du sujet ", dans H. DREYFus et P. RABINOW, Michel Foucault. Un parcours philosophique, Paris, Gallimard, 1984, pp. 297-308.

19. Michel FouCAULT, " Omnes et singulatim », p. 17.

20. Ibidem, p. 19. 
1951, Paul Ricoeur signalait que la question de l'autorité (et donc du pouvoir) n'est pas accidentelle à la théologie puisque l'autorité de la Parole de Dieu pour le croyant constitue un aspect fondamental de la Révélation. Ricoeur dénonçait la violence liée « au pouvoir clérical du vrai » et à l'enchaînement " autorité du Verbe, autorité du témoignage scripturaire, autorité de la prédication fidèle, autorité de la théologie. ${ }^{21}$ Or, cet enchaînement pose un problème particulier à la théologie dans la mesure où s'y joue non seulement l'autorité de Dieu sur le croyant, mais celle de l'homme sur l'homme, sous couvert de la Parole de Dieu. Relevant du « magistère authentique » ou « autorisé », l'interprétation est alors au service du pouvoir dominant.

Mais cela n'est peut-être pas aussi tranché. En effet, l'autorité religieuse comme les totalitarismes politiques ou politico-religieux repoussent d'emblée toute interprétation divergente, voire toute interprétation puisque, selon leurs dires, "la vérité n'est pas une question d'interprétation ". Dans un tel contexte, l'herméneutique apparaît comme l'arme des sans-pouvoir. Qu'a-t-on besoin d'herméneutique, qu'a-t-on besoin de se réclamer d'herméneutique quand on a le pouvoir d'imposer sa vérité? "Ce sont les demandeurs qui interprètent, dit-il; ceux qui ont et qui sont déclarent ${ }^{22}$ ». Voilà peut-être pourquoi Starobinski affirmait que l'acte d'interprétation porte un potentiel révolutionnaire, comme le rappelle Walter Moser dans sa note sur «Pouvoir et interprétation ». On n'en saisit peut-être que mieux l'importance que la théologie féministe accorde au pouvoir de dire, de nommer, de définir le monde et de raconter l'histoire, y compris l'histoire sainte.

\section{Une question théologique majeure}

Construite sur une expérience religieuse et comme démarche critique face à celle-ci, la théologie a entretenu un rapport trouble avec le pouvoir. La louange du pouvoir divin, dont participaient les pouvoirs de ce monde, a conduit la théologie de l'Empire à faire l'apologie du pouvoir des puissants, tout en étant ici et là critique à son endroit. Plus récemment, la théologie est massivement passée de cette apologie à un soup-

21. Paul Ricoeur, "Vérité et mensonge ", Histoire et vérité, Paris, Seuil, 1955, p. 180.

22. Michelet, cité dans Michel DESPLAND, « Un tournant vers l'herméneutique en France en 1806?", SR, Vol. 23, no.1 (1994) p. 17, n. 64. 
çon généralisé du pouvoir saisi comme domination. Même les tenants du pouvoir ecclésiastique ont remplacé une théologie du pouvoir par une théologie du service, mais plusieurs jugent qu'il ne s'agit là que d'un discours occultant l'exercice autarcique du pouvoir ministériel. D'où la valeur du titre apparemment naiff de l'article de Jacques Racine : "Le pouvoir existe ».

Si l'analyse et la critique de l'exercice du pouvoir ecclésial ont longtemps échappé à la théologie, plus soucieuse de sa divine origine, il en va autrement aujourd'hui. De concert avec les autres sciences humaines, mais avec ses outils propres, la théologie critique vivement l'exploitation religieuse du pouvoir divin. Cette conversion du discours théologique sur le pouvoir émerge en partie des requêtes de l'existence croyante et de la critique des autorités portée par le Nazaréen. Elle lui vient aussi de la culture et du paradigme empirico-historique plutôt qu'essentialiste des sciences sociales qui lui ont révélé l'usage abusif du pouvoir divin ou, plus précisément, du discours sur celui-ci. La théologie a alors, en maints lieux, effectué un renversement de son herméneutique du pouvoir et s'est tournée davantage vers le point de vue des victimes ou des sans-pouvoir que vers celui des puissants.

D'une part, donc, la théologie critique, avec les sciences sociales comme avec l'Évangile, les usages humains du pouvoir divin. D'autre part, elle porte sa critique jusqu'au concept même du pouvoir divin, comme on le verra dans les articles de Louise Melançon, Rosemary Ruether et Jean Richard. En nombre de cas, le centre discursif de la théologie s'est même inversé, passant du Pantocrator au Crucifié, voire du Père tout-puissant au Fils victime.

En fait, le pouvoir divin a toujours constitué un sujet de réflexion majeur pour la théologie chrétienne. Dès les débuts, devant la croix de Jésus puis devant la souffrance et le mal, les croyants se sont trouvé et se trouvent toujours - confrontés de façon radicale à la question du pouvoir de Dieu. Mais l'étonnement et les questions ont été largement occultés par les exigences d'affirmations définitives de la liturgie, de la dogmatique et de la catéchèse. C’est ainsi que la métaphore du Seigneur Dieu est si répandue en Occident qu'elle passe pour dire la réalité crue $^{23}$. Or, cette image d'un monarque absolu régnant sur son royaume

23. J'aime bien le paradoxe de cette " réalité crue ». 
supports conceiving of God as a being existing somewhere apart from the world and ruling it externally either directly through divine intervention or indirectly through controlling the wills of his subjects. [...] The understanding of salvation that accompanies this view is sacrificial, substitutionary atonement, and in Anselm's classic rendition of it the sovereign imagery predominates. Since even a wink of the eye by a vassal against the Liege Lord of the universe would be irredeemable sin, we as abject subjects must rely totally on our sovereign God who'became man' in order to undergo a sacrificial death, substituting his great worth for our worthlessness. Again, we feel the power of this picture : because we are totally unable to help ourselves, we will be totally cared for. We not only are forgiven for our sins and reconciled to our King as once again his loyal subjects but we can also look forward to a time when we shall join him in his heavenly kingdom. ${ }^{24}$

Comme le signale McFague elle-même, ces commentaires semblent relever de la caricature, d'autant que l'image du Seigneur n'est pas la seule image de Dieu portée par la tradition chrétienne. Mais voilà que ces autres images, particulièrement celle du Père, ont été capturées dans l'orbite du modèle monarchique. "The model of God as father [...] could have gone in the direction of parent (and that is clearly its New Testament course), with its associations of nurture, care, guidance, concern, and self-sacrifice, but under the powerful influence of the monarchical model, the parent became the patriarch, and patriarchs act more like kings than like fathers : they rule their children and they demand obedience $^{25}$ ». Le même sort a échu au concept réformé de la Parole, comprise comme "Parole du Seigneur ». Le problème n'est pas seulement théologique, mais bien pratique dans la mesure où ce modèle monarchique a justifié sinon nourri non seulement de nombreux pouvoirs mais aussi et surtout de nombreuses oppressions.

Le Psalm of Anger to a Patriarchal god ${ }^{26}$ illustre de vive façon quelques-unes des conséquences d'un tel modèle et de son usage politique

24. Sallie McFague, Models of God, Philadelphia, Fortress, 1987, p. 64.

25. Ibid., p. 66. On pourrait toutefois se demander si l'image du patriarche n'a pas plutôt devancé l'autre.

26. On pourra juger particulièrement significative la graphie de "god" avec la minuscule. Le Psalm est de Sheila A. Redmond, "Confrontation Between the Christian God and an Abused Child 25 Years Later », dans Family Violence in a Patriarchal Society: A Challenge to the Church, Ottawa, 
ou, en ce cas, domestique. Suite à une discussion que nous avions eue, Rosemary Ruether l'évoque au début de son article, mais pour se distancier aussitôt de la figure de Dieu (ou de dieu) qu'il met en scène et en proposer plutôt une tout autre. Ce Psaume troublant a été créé pour une liturgie dans le cadre d'une session œcuménique sur la violence faite aux femmes. Malgré ses critiques radicales, il s'identifie comme un psaume, une prière, et il l'est, comme le manifeste l'appel déchirant de sa conclusion : "Où étais-Tu quand j'avais besoin de Toi? » Les familiers des Psaumes et surtout du livre de Job y reconnaîtront une dynamique biblique où Dieu est pris à partie par le souffrant. James Poling, psychothérapeute avec des survivantes d'abus sexuels, y voit une œuvre courageuse qui correspond à un stade que traversent la plupart des survivantes. Tout en signalant qu'il faut rejeter les images abusives de Dieu que le psaume dénonce, il se demande comment nous pouvons articuler l'expérience dont témoigne ce Psaume avec l'expérience que des survivantes font aussi de la grâce divine ${ }^{27}$. On pourra dire que les visions théologiques qu'il met en scène sont fausses, tronquées ou perverties. Or, c'est justement en cela qu'il illustre quelques-unes des aberrations auxquelles a pu mener une certaine éducation chrétienne conjuguée à des notions déficientes de la puissance divine, des rapports homme/ femme, de la sexualité, de l'obéissance ou de la souffrance, etc ${ }^{28}$.

La question du pouvoir traverse toute la théologie comme elle fait des sciences humaines et la remise en cause du pouvoir divin concerne non seulement la théologie mais toute l'existence chrétienne, de la liturgie jusqu'à l'éthique. Si métaphysique puisse-t-elle paraître, la question théologique du pouvoir divin n'est pas étrangère à l'expérience quotidienne. Déterminant la figure de la divinité, elle détermine l'existence des croyants qui s'y réfèrent pour orienter leur vie et y faire sens. Elle concerne la façon dont les croyants sont appelés à construire leur rapport au pouvoir, c'est-à-dire à eux-mêmes, aux autres et à la vie ellemême.

Justice and Corrections, Family Violence in a Patriarchal Culture: A Challenge to Our Way of Living, Ottawa, CCJC : CCSD, 1988, p. 118.

27. Correspondance personnelle avec James POLING, printemps 2000.

28. Nous avons présenté certaines de ces conséquences, de même que traduit le Psalm of Anger, avec la permission de l'auteure, dans Jean-Guy NADEAU, "Éducation chrétienne et réaction à l'inceste ", Prêtre et Pasteur, vol 94, no 5, (mai 1991) 276-286. 